\title{
Stability Monitoring of Batch Processes with Iterative Learning Control
}

\author{
Yan Wang, Junwei Sun, Taishan Lou, and Lexiang Wang \\ College of Electronic and Information Engineering, Zhengzhou University of Light Industry, Zhengzhou 450002, China \\ Correspondence should be addressed to Yan Wang; wyan@zzuli.edu.cn
}

Received 14 November 2016; Accepted 5 January 2017; Published 31 January 2017

Academic Editor: Xavier Leoncini

Copyright (C) 2017 Yan Wang et al. This is an open access article distributed under the Creative Commons Attribution License, which permits unrestricted use, distribution, and reproduction in any medium, provided the original work is properly cited.

\begin{abstract}
In recent years, the iterative learning control (ILC) is widely used in batch processes to improve the quality of the products. Stability is a preoccupation of batch processes when the ILC is applied. Focusing on the stability monitoring of batch processes with ILC, a method based on innerwise matrix with considering the uncertainty of the model and disturbance was proposed. First, the batch process with ILC was derived as a two-dimensional autoregressive and moving average (2D-ARMA) model. Then two kinds of stability indices are constructed based on the innerwise matrix through the identification of the 2D-ARMA. Finally, the statistical process control (SPC) chart was adopted to monitor those stability indices. Numerical results are presented to demonstrate the effectiveness of the proposed method.
\end{abstract}

\section{Introduction}

Batch processes are the main way to produce the high value-added products such as fine chemicals industry and biopharming. However, in batch processes, sensor variables will exhibit strong within-batch autocorrelation as well as nontrivial batch-to-batch correlations [1]. Essentially, batch processes with dynamics along both time and batch directions are referred to as a two-dimensional (2D) system. The design of controller in batch processes is different from that in continuous ones. There are many ways to make sure of the stabilization of a feedback control loop when the process is operated continuously. Since the existence of 2D dynamic, the conventional feedback controller for continuous processes may not be effective for batch processes [2].

Recently, the iterative learning control (ILC) has received tremendous attention in batch processes [2]. It is a general technique used to improve the control performance of a system by refining control input from cycle to cycle. ILC of batch processes improves the quality of products by considering the use of previous batch information to control the current one. It not only does make sure of the system stabilization of every batch operation by using feedback controller, but also improves the tracking performance by using feedforward controller.
Since the controller performance is closely related to the quality and product yield of industrial products, some scholars have analyzed the ILC controller performance of batch processes. Chen and Kong introduced an optimal ILC index to evaluate the controller performance based on minimum variance [2]. Wei et al. proposed a new controller performance analysis (CPA) method based on linear quadratic Gaussian ILC under the condition that the system model is unknown [3]. However, the above methods focused mainly on the performance assessment of ILC controller. They did not study the stability of batch processes. Stability is a preoccupation of batch processes when the ILC is applied. The degradation of controller performance, actuator breakdown, or the unexpected interference all can make system stabilization worsen. Shi et al. defined the concept of batch convergence with considering the unknown disturbance and initial value [4]. Wang et al. introduced an ILC-PI controller and analyzed the robust asymptotically stable conditions of batch processes [5]. However, those stability research papers focused mainly on analyzing the concept of batch convergence. They did not show how to monitor the stability of the batch operation. Because of the intrinsically dynamic operations of batch processes, some stability problems still go undiagnosed for a prolong period of time. In this paper, stability monitoring of batch operation system with ILC is 
developed. Because of the large variation of batch processes in the operation condition during a batch run, the stability index during a batch run came into place for online stability monitoring. Siljak came up with a $2 \mathrm{D}$ system stability test method based on the Schwarz model and innerwise matrix [6]. Based on their methods, the paper proposed two kinds of stability indices along both time and batch directions.

Based on the 2D system theory, the closed-loop batch processes with ILC can be expressed 2D time series model [7]. The proposed method adopted a general 2D-ARMA model to express the ILC batch process with considering the uncertainty of the model and disturbance. However, it is not easy to identify that time series under close-loop conditions. Since there are many terms available in a 2D-ARMA, an effective fault monitoring method can be developed only if accurate 2D model identification is available. Aksasse and Radouane adopted the 2D Akaike information criterion (AIC) to identify the autoregressive model [8]. Yao and Gao proposed an automatic determination method based on the iterative stepwise regressions (SWR) to identify the $2 \mathrm{D}$ batch processes model and obtained a better identify result [9]. Recently, an adaptive least absolute shrinkage and selection operator (LASSO) had become one of the most popular approaches for automatic variable selection in linear regression [10]. Wang et al. compared the advantage and disadvantage of adaptive LASSO and SWR algorithm [11]. They indicated that the adaptive LASSO procedure in terms of order estimation is much better than the SWR and the SWR is slightly superior in terms of accuracy in values of true coefficients. The paper considered the advantage of those two methods. An integrated method of adaptive LASSO and SWR was adopted to identify the orders and coefficients of that 2D time series. Compared with the existing methods, the integrated method has better identification performance. Given the coefficients and orders of the identified model, the stability indices can be easily computed and monitored by SPC chart.

The rest of this paper is organized as follows: Section 2 will introduce the 2D-ARMA model and give the estimation of white noise. Section 3 will introduce our methodology including the derivation of the 2D-ARMA model of the batch process with ILC, the construction of innerwise matrix, and the definition of the stability indices. The effectiveness of the proposed approach is demonstrated in Section 4 by some simulations. The last section gives our conclusions.

\section{2D-ARMA Model}

The 2D-ARMA model of a batch process with considering the influence of disturbance can be given by the following model [12]:

$$
\begin{aligned}
y(i, k)= & \underbrace{\sum_{m=0}^{M} \sum_{n=0}^{N} a_{m n} y(i-m, k-n)}_{m, n \neq 0,0} \\
& +\underbrace{\sum_{p=0}^{P} \sum_{q=0}^{Q} b_{p q} \varepsilon(i-p, k-q)+\varepsilon(i, k),}_{p, q \neq 0,0}
\end{aligned}
$$

where $i$ represents the within-batch time index, $k$ represents the batch-to-batch index, $a_{m n}$ is the coefficient of the autoregressive (AR) part, and $b_{p q}$ is the coefficient of the moving average (MA) part. $\varepsilon(i, k) \sim N\left(0, \sigma^{2}\right)$ is an $2 \mathrm{D}$ unobserved random white noise. $M, N, P, Q$ are the orders of the $2 \mathrm{D}$ ARMA model, respectively.

To estimate the value of $\varepsilon(i, k)$, we adopt a $2 \mathrm{D}$ long $\mathrm{AR}$ model to express the output $y(i, k)$ of the batch system [11]:

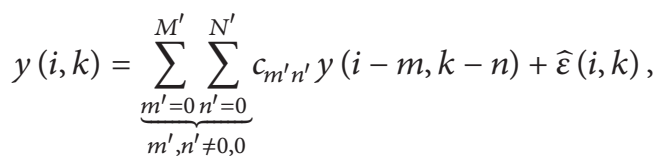

where $M^{\prime}, N^{\prime} \gg M, N$ and the coefficient $c_{m^{\prime} n^{\prime}}$ and $\widehat{\varepsilon}(i, k)$ of the above formulation can be obtained by linear regression.

The 2D-ARMA model (1) can be also considered as a discrete system with transfer function [13]:

$$
H\left(z_{1}^{-1}, z_{2}^{-1}\right)=\frac{B\left(z_{1}^{-1}, z_{2}^{-1}\right)}{A\left(z_{1}^{-1}, z_{2}^{-1}\right)}=\frac{\sum_{p=0}^{P} \sum_{q=0}^{\mathrm{Q}} b_{p q} z_{1}^{-p} z_{2}^{-q}}{\sum_{m=0}^{M} \sum_{n=0}^{N} a_{m n} z_{1}^{-m} z_{2}^{-n}}
$$

where $z_{1}^{-1}\left(z_{1}^{-1} y(i, k)=y(i-1, k)\right)$ is the back-shift operator for the within-batch time index $i$ and $z_{2}^{-1}\left(z_{2}^{-1} y(i, k)=y(i, k-\right.$ $1)$ ) is the back-shift operator for the batch-to-batch index.

\section{The Stability Monitoring Scheme}

3.1. The Batch Process with ILC. Consider a SISO system with performing repetitively an operation in a limited time $T$, called a batch run $k$. The feedback control structure of the batch system is shown in Figure 1. From Figure 1, the output of the batch process can be expressed as follows [2]:

$$
\begin{aligned}
y(i, k)=G_{p}\left(z_{1}^{-1}, k\right) u(i, k) & +G_{\varepsilon}\left(z_{1}^{-1}, k\right) \varepsilon(i, k) \\
i & =1,2, \ldots, T ; k=1,2, \ldots,
\end{aligned}
$$

where $T$ is the running time of each batch, $G_{p}\left(z_{1}^{-1}, k\right)$ and $G_{\varepsilon}\left(z_{1}^{-1}, k\right)$ are the process and disturbance transfer function, respectively, and $u(i, k)$ is the controller output.

According to the feedback control structure of each batch $k$, the controller output $u(i, k)$ can be expressed as

$$
u(i, k)=G_{c}\left(z_{1}^{-1}, k\right)\left(x^{\mathrm{sp}}(i, k)-y(i, k)\right)
$$

where $x^{\mathrm{sp}}(i, k)$ is the reference signal and $G_{c}\left(z_{1}^{-1}, k\right)$ is the feedback controller.

Substituting (5) into (4), the output can be expressed as

$$
\begin{aligned}
y(i, k)= & \frac{G_{p}\left(z_{1}^{-1}, k\right) G_{c}\left(z_{1}^{-1}, k\right)}{1+G_{p}\left(z_{1}^{-1}, k\right) G_{c}\left(z_{1}^{-1}, k\right)} x^{\mathrm{sp}}(i, k) \\
& +\frac{G_{\varepsilon}\left(z_{1}^{-1}, k\right)}{1+G_{p}\left(z_{1}^{-1}, k\right) G_{c}\left(z_{1}^{-1}, k\right)} \varepsilon(i, k) .
\end{aligned}
$$




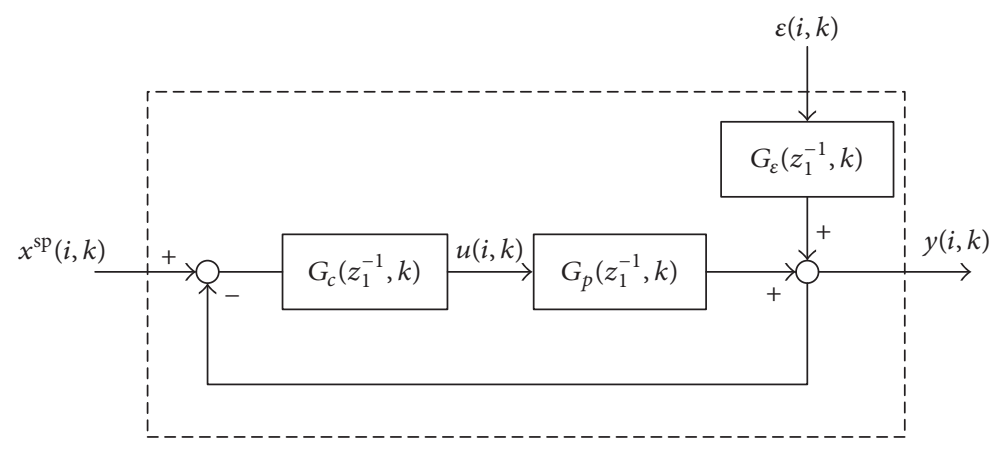

FIGURE 1: The feedback control structure of each batch $k$

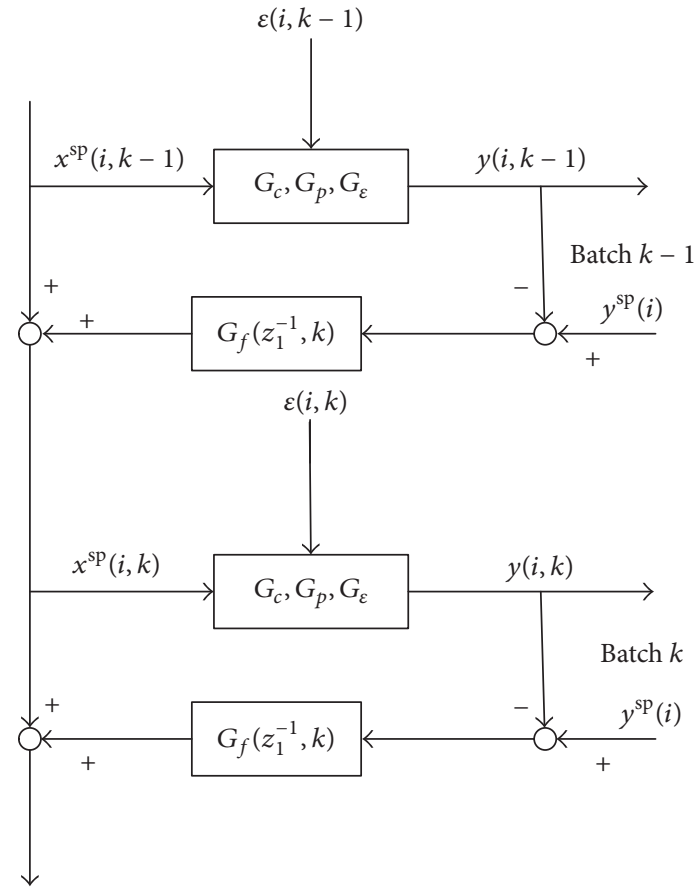

FIGURE 2: The schematics of ILC with the feedback controller $\left(G_{c}\left(z_{1}^{-1}, k\right)\right)$ and the feedforward controller $\left(G_{f}\left(z_{1}^{-1}, k\right)\right)$.

The principle of ILC adopts the previous batch output $y(i, k-1)$ and the reference signal $x^{\mathrm{sp}}(i, k-1)$ to control during the current batch $k$ under the disturbance input $\varepsilon(i, k)[2]$. The specific ILC structure is shown in Figure 2, and the update law can be expressed as follows:

$$
\begin{aligned}
x^{\mathrm{sp}}(i, k)= & x^{\mathrm{sp}}(i, k-1) \\
& +\left(y^{\mathrm{sp}}(i)-y(i, k-1)\right) G_{f}\left(z_{1}^{-1}, k\right),
\end{aligned}
$$

where $y^{\mathrm{sp}}(i)$ is the expected output of each batch and $G_{f}\left(z_{1}^{-1}, k\right)$ is the feedforward controller and adjusts the reference signal $x^{\mathrm{sp}}(i, k)$ by the output errors $e(i, k-1)=$ $y^{\mathrm{sp}}(i)-y(i, k-1)$.
Since $z_{2}^{-1} y(i, k)=y(i, k-1)$, the modified desired trajectory $x^{\mathrm{sp}}(i, k)$ is given as

$$
x^{\mathrm{sp}}(i, k)=\frac{G_{f}\left(z_{1}^{-1}, k\right)}{1-z_{2}^{-1}} e(i, k-1) .
$$

Substituting (8) into (6), the following equation can be obtained:

$$
\begin{aligned}
(1+ & \left.G_{p}\left(z_{1}^{-1}, k\right) G_{c}\left(z_{1}^{-1}, k\right)\right)(y(i, k)-y(i, k-1)) \\
= & G_{p}\left(z_{1}^{-1}, k\right) G_{c}\left(z_{1}^{-1}, k\right) G_{f}\left(z_{1}^{-1}, k\right) e(i, k-1) \\
& +G_{\varepsilon}\left(z_{1}^{-1}, k\right)(\varepsilon(i, k)-\varepsilon(i, k-1)) .
\end{aligned}
$$

By adding and subtracting the setpoint $y^{\mathrm{sp}}(i),(9)$ can also be written as

$$
\begin{aligned}
(1 & \left.+G_{p}\left(z_{1}^{-1}, k\right) G_{c}\left(z_{1}^{-1}, k\right)\right) \\
& \cdot\left(y(i, k)-y^{\mathrm{sp}}(i)-\left(y(i, k-1)-y^{\mathrm{sp}}(i)\right)\right) \\
& =G_{p}\left(z_{1}^{-1}, k\right) G_{c}\left(z_{1}^{-1}, k\right) G_{f}\left(z_{1}^{-1}, k\right) e(i, k-1) \\
& +G_{\varepsilon}\left(z_{1}^{-1}, k\right)(\varepsilon(i, k)-\varepsilon(i, k-1)) .
\end{aligned}
$$

So the error $e(i, k)$ can be written as a function of the previous batch error $e(i, k-1)$ :

$$
\begin{aligned}
(1 & \left.+G_{p}\left(z_{1}^{-1}, k\right) G_{c}\left(z_{1}^{-1}, k\right)\right) e(i, k)=(1 \\
& +G_{p}\left(z_{1}^{-1}, k\right) G_{c}\left(z_{1}^{-1}, k\right) \\
& \left.-G_{p}\left(z_{1}^{-1}, k\right) G_{c}\left(z_{1}^{-1}, k\right) G_{f}\left(z_{1}^{-1}, k\right)\right) e(i, k-1) \\
& -G_{\varepsilon}\left(z_{1}^{-1}, k\right)(\varepsilon(i, k)-\varepsilon(i, k-1)) .
\end{aligned}
$$


According to Box et al's study [12], the above relation can be transformed into the following 2D-ARMA form:

$$
\begin{aligned}
& G\left(z_{1}^{-1}\right) e(i, k)= H\left(z_{1}^{-1}\right) e(i, k-1)-N\left(z_{1}^{-1}\right) \varepsilon(i, k) \\
&+N\left(z_{1}^{-1}\right) \varepsilon(i, k-1) \\
& G\left(z_{1}^{-1}\right)= 1+g_{1} z_{1}^{-1}+g_{2} z_{1}^{-2}+\cdots+g_{r} z_{1}^{-r} \\
& H\left(z_{1}^{-1}\right)=1+h_{1} z_{1}^{-1}+h_{2} z_{1}^{-2}+\cdots+h_{s} z_{1}^{-s} \\
& N\left(z_{1}^{-1}\right)=1+n_{1} z_{1}^{-1}+n_{2} z_{1}^{-2}+\cdots+n_{v} z_{1}^{-v},
\end{aligned}
$$

where $r, s, v$ are the orders of $G\left(z_{1}^{-1}\right), H\left(z_{1}^{-1}\right), N\left(z_{1}^{-1}\right)$, respectively.

By moving $H\left(z_{1}^{-1}\right) e(i, k-1)$ term to the left, (12) can be written as

$$
\begin{aligned}
& \left(G\left(z_{1}^{-1}\right)-H\left(z_{1}^{-1}\right) z_{2}^{-1}\right) e(i, k) \\
& \quad=-N\left(z_{1}^{-1}\right)\left(1-z_{2}^{-1}\right) \varepsilon(i, k) .
\end{aligned}
$$

And the ILC batch process can be considered as a discrete system with transfer function:

$$
\begin{aligned}
H\left(z_{1}^{-1}, z_{2}^{-1}\right) & =-\frac{N\left(z_{1}^{-1}\right)\left(1-z_{2}^{-1}\right)}{G\left(z_{1}^{-1}\right)-H\left(z_{1}^{-1}\right) z_{2}^{-1}} \\
& =\frac{B\left(z_{1}^{-1}, z_{2}^{-1}\right)}{A\left(z_{1}^{-1}, z_{2}^{-1}\right)}
\end{aligned}
$$

3.2. Stability Analysis of Batch Process with ILC. According to the definition of bounded-input bounded-output (BIBO) stable, the $1 \mathrm{D}$ system is BIBO stable if all zeros of $A\left(z_{1}^{-1}, 0\right)$ are not more than the unit circle. Consequently, the $2 \mathrm{D}$ polynomial $A\left(z_{1}^{-1}, z_{2}^{-1}\right)$ is said to be BIBO stable if [6]

$$
\begin{aligned}
A\left(z_{1}^{-1}, 0\right) \neq 0, & \left|z_{1}^{-1}\right| \leq 1, \\
A\left(z_{1}^{-1}, z_{2}^{-1}\right) \neq 0, & \left|z_{1}^{-1}\right|=1,\left|z_{2}^{-1}\right| \leq 1 .
\end{aligned}
$$

Let us write $A\left(z_{1}^{-1}, z_{2}^{-1}\right)$ as a polynomial in $z_{2}^{-1}$ with coefficients that are polynomials in $z_{1}^{-1}$ :

$$
\begin{aligned}
A\left(z_{1}^{-1}, z_{2}^{-1}\right) & =\sum_{n=0}^{N} d_{N-n}\left(z_{1}^{-1}\right) z_{2}^{-n} \\
d_{N-n}\left(z_{1}^{-1}\right) & \equiv \sum_{m=0}^{M} a_{m n} z_{1}^{-m} .
\end{aligned}
$$

In the pioneering paper [14], the concept of innerwise matrix has been used to analyze the stability of single dimensional systems. Kanellakis et al. extended it to 2D case and created the following innerwise matrix $\Delta_{2 N}\left(z_{1}^{-1}\right)[13]$ :

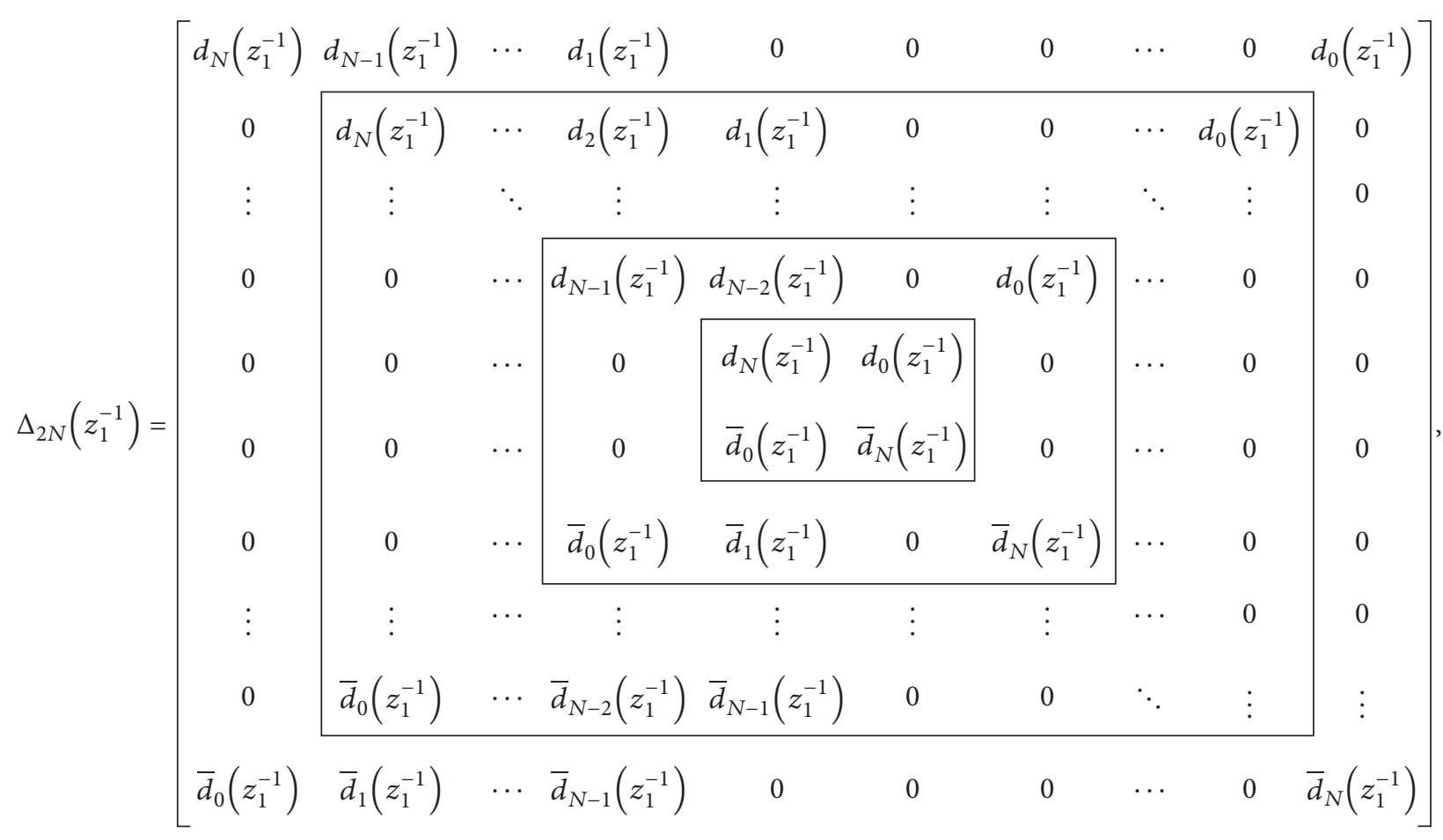

where $\bar{d}_{N}\left(z_{1}^{-1}\right)$ is the complex conjugate of $d_{N}\left(z_{1}^{-1}\right)$. 
According to (16) and (17), the innerwise matrix $\Delta_{2}\left(z_{1}^{-1}\right)$ of batch process with ILC can be constructed as

$$
\Delta_{2}\left(z_{1}^{-1}\right)=\left[\begin{array}{ll}
d_{1}\left(z_{1}^{-1}\right) & d_{0}\left(z_{1}^{-1}\right) \\
\bar{d}_{0}\left(z_{1}^{-1}\right) & \bar{d}_{1}\left(z_{1}^{-1}\right)
\end{array}\right],
$$

where $d_{1}\left(z_{1}^{-1}\right) \equiv G\left(z_{1}^{-1}\right)=1+g_{1} z_{1}^{-1}+\cdots+g_{r} z_{1}^{-r}$ and $d_{0}\left(z_{1}^{-1}\right) \equiv$ $H\left(z_{1}^{-1}\right)=h_{0}+h_{1} z_{1}^{-1}+\cdots+h_{s} z_{1}^{-s}$.

Siljak pointed that, for positivity checking of such an innerwise matrix, one requires the positivity checking of the matrix at one point, say at $z=1$, and the positivity checking of the determinant for all $|z|=1$ [6]. Based on their study, we proposed two indices to analyze the stability of batch process with ILC:

$$
\begin{aligned}
& R 1=\text { max_positive_root_of }\left[A\left(z_{1}^{-1}, 0\right)=0\right] \leq 1, \\
& R 2=\text { determination }\left[\Delta_{2}\left(z_{1}^{-1}\right)\right] \geq 0 \\
& \qquad \forall z_{1}^{-1},\left|z_{1}^{-1}\right|=1,
\end{aligned}
$$

where $R 1$ is the stability index in the within-batch time direction and $R 2$ is the stability index in the batch-to-batch direction. The system will not be stable if the within-batch dynamic is not stable. Even the system is stable in the withinbatch time direction if (19) is valid, the system can be unstable in the batch-to-batch if (19) and (20) are violated.

3.3. The Process of Stability Monitoring Scheme. In order to ensure the safe operation of batch processes with ILC, the paper proposed a real-time stability monitoring method, which contains two phases. Phase 1 is to establish control limits in normal period for each stability index. Phase 2 is to monitor each stability index online and to determine each stability index in control. The details are shown as follows.

Phase 1. (1) Collect the dynamic batch processes data in a normal period.

(2) Identify the 2D-ARMA model for each batch in the normal period by adopting an adaptive LASSO algorithm to identify the order of 2D-ARMA model and an SWR algorithm to determine the coefficients of 2D-ARMA model.

(3) Construct the innerwise matrix $\Delta_{2}\left(z_{1}^{-1}\right)$ based on the identified coefficients of 2D-ARMA model and calculate the stability index $R 1$ and $R 2$.

(4) Establish Shewhart control charts for each stability indices.

Phase 2. (1) Collect the dynamic batch processes data at the current batch $k$.

(2) Identify the 2D-ARMA model for this batch $k$ by adopting adaptive LASSO and SWR algorithm.

(3) Construct the innerwise matrix $\Delta_{2}\left(z_{1}^{-1}\right)$ and calculate the stability indices $R 1$ and $R 2$ for this batch $k$.

(4) Determine whether $R 1$ and $R 2$ for this batch $k$ are in control.

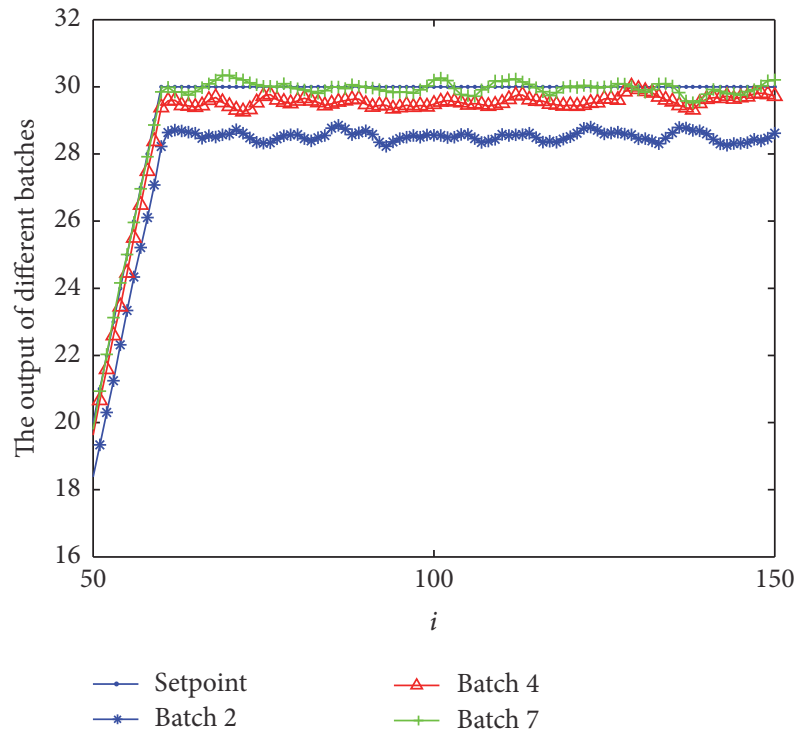

FIgURE 3: The controlled output.

\section{Simulation}

A batch process is given as follows:

$$
\begin{aligned}
G_{p} & =\frac{0.6-0.3 z^{-1}}{1-0.7 z^{-1}}, \\
G_{\varepsilon} & =\frac{z^{-2}}{1-1.6 z^{-1}+0.63 z^{-2}} .
\end{aligned}
$$

The feedback and feedforward controllers are $G_{c}=1 /(1-$ $0.9 z^{-1}$ ) and $G_{f}=0.5$. Under those two controllers, the process can track the setpoint after seven batches, which is shown in Figure 3. In subsequent monitoring, a window with 30 batches, each batch has 1000 data samples and is used to estimate the 2D-ARMA model. Seventy moving windows are created by moving the window of 30 batches forward one batch at a time. The first 30 windows are used as "normal" data to estimate the control limits. The limits UCL $=\mu+3 \sigma$ and LCL $=\mu-3 \sigma$ are the conventional control limits, which are widely used in SPC. Variations beyond these two limits indicate that there are substantial changes to the process. The system become unstable as variations exceeded the stability limits $\mathrm{SL}=1$ or $\mathrm{SL}=0$. The within-batch dynamic is unstable as the value of $R 1$ is greater than 1 and the batch-to-batch dynamic is unstable as the value of $R 2$ is smaller than 0 . Two step changes $G_{p}=\left(0.6-0.3 z^{-1}\right) /\left(1-0.7 z^{-1}\right) \rightarrow G_{p}=(0.45-$ $\left.0.3 z^{-1}\right) /\left(1-0.7 z^{-1}\right)$ and $G_{p}=\left(0.6-0.3 z^{-1}\right) /\left(1-0.7 z^{-1}\right) \rightarrow$ $G_{p}=\left(0.6 z^{-1}-0.3 z^{-2}\right) /\left(1-0.7 z^{-1}\right)$ have been introduced at 31st window. Figures 4 and 5 show the output of different batches when those two step changes happened, respectively. It can be clearly see that the system is unstable when there is a process change $G_{p}=\left(0.6-0.3 z^{-1}\right) /\left(1-0.7 z^{-1}\right) \rightarrow G_{p}=$ $\left(0.6 z^{-1}-0.3 z^{-2}\right) /\left(1-0.7 z^{-1}\right)$. 


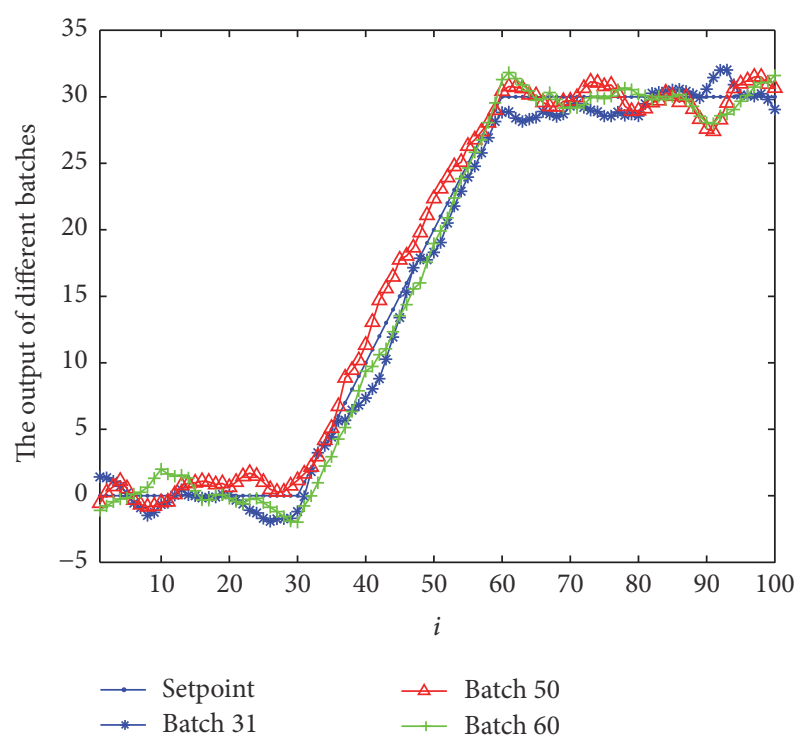

FIGURE 4 : The controlled output with a process change $G_{p}=(0.6-$ $\left.0.3 z^{-1}\right) /\left(1-0.7 z^{-1}\right) \rightarrow G_{p}=\left(0.45-0.3 z^{-1}\right) /\left(1-0.7 z^{-1}\right)$.

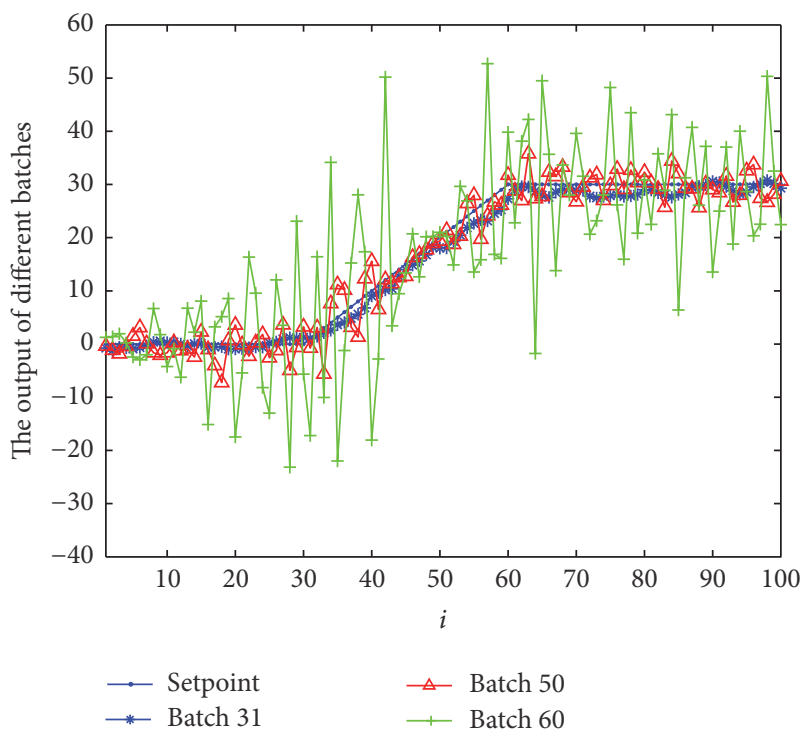

FIGURE 5: The controlled output with a process change $G_{p}=(0.6-$ $\left.0.3 z^{-1}\right) /\left(1-0.7 z^{-1}\right) \rightarrow G_{p}=\left(0.6 z^{-1}-0.3 z^{-2}\right) /\left(1-0.7 z^{-1}\right)$.

Figure 6 shows the stability monitoring with a process change $G_{p}=\left(0.6-0.3 z^{-1}\right) /\left(1-0.7 z^{-1}\right) \rightarrow G_{p}=$ $\left(0.45-0.3 z^{-1}\right) /\left(1-0.7 z^{-1}\right)$. From Figure 6 , it can be clearly see that those two indices are not more than the stability limit although they are beyond the control limit at the 35th window, which indicated that the within-batch process and batch-to-batch dynamics are still stable. Figure 7 shows the stability monitoring with a process change $G_{p}=(0.6-$ $\left.0.3 z^{-1}\right) /\left(1-0.7 z^{-1}\right) \rightarrow G_{p}=\left(0.6 z^{-1}-0.3 z^{-2}\right) /\left(1-0.7 z^{-1}\right)$. From Figure 7, $R 1$ had become smaller when the process change occurred, which indicated that within-batch process

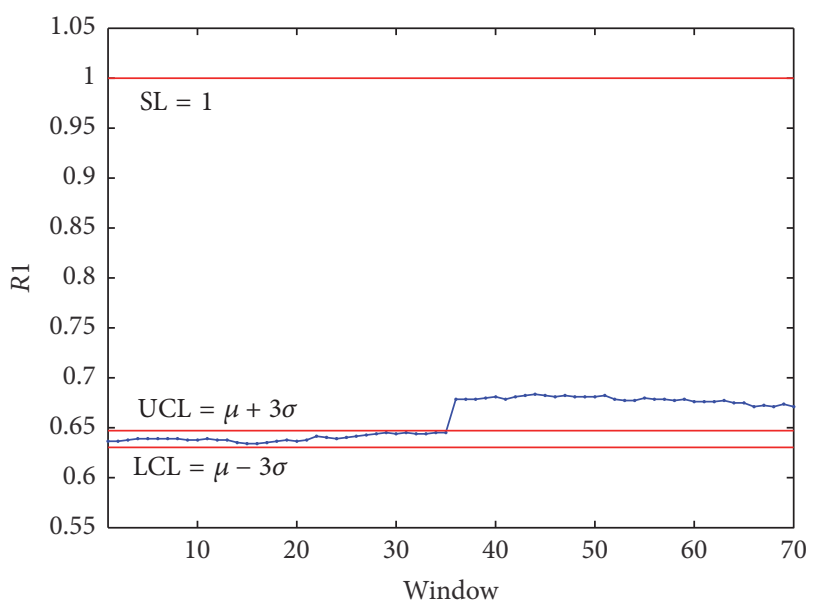

(a)

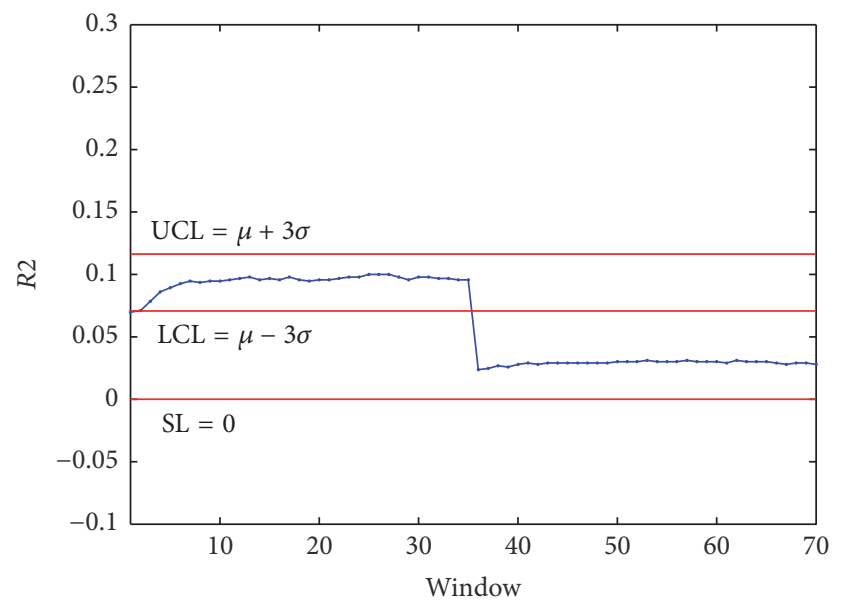

(b)

FIgURE 6: Stability monitoring with a process change $G_{p}=(0.6-$ $\left.0.3 z^{-1}\right) /\left(1-0.7 z^{-1}\right) \rightarrow G_{p}=\left(0.45-0.3 z^{-1}\right) /\left(1-0.7 z^{-1}\right)$.

is still stable. However, $R 2$ was beyond the stability limit $\mathrm{SL}=$ 0 , which indicated that the batch-to-batch dynamics suffer a substantial change leading to system instability.

\section{Conclusion}

In this paper, the batch process with ILC was derived as a 2DARMA model. An integrated technology of adaptive LASSO and SWR was adopted to identify the 2D-ARMA model. The paper analyzed the stability of ILC batch process based on innerwise matrix and proposed two stability indices. Finally, conventional SPC chart was used to monitor those stability indices. Some simulation results showed the effectiveness of the proposed method.

\section{Competing Interests}

The authors declare that they have no competing interests. 


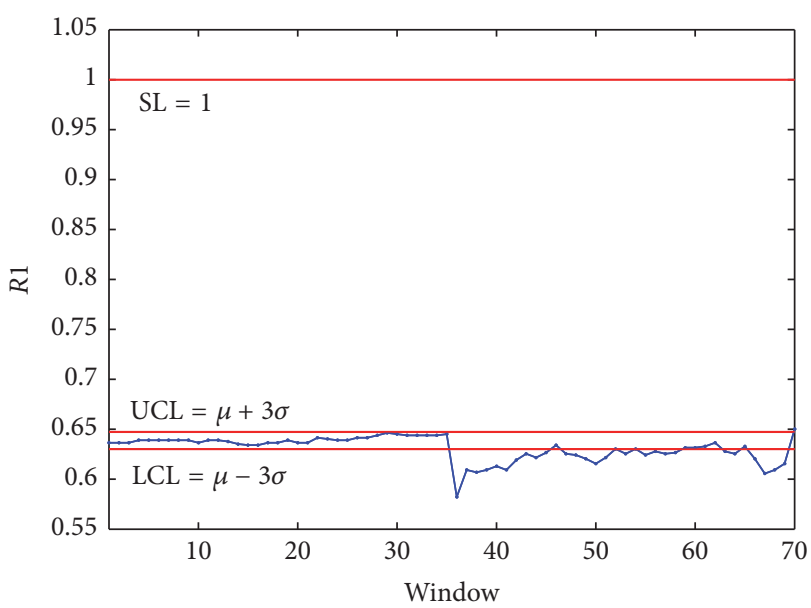

(a)

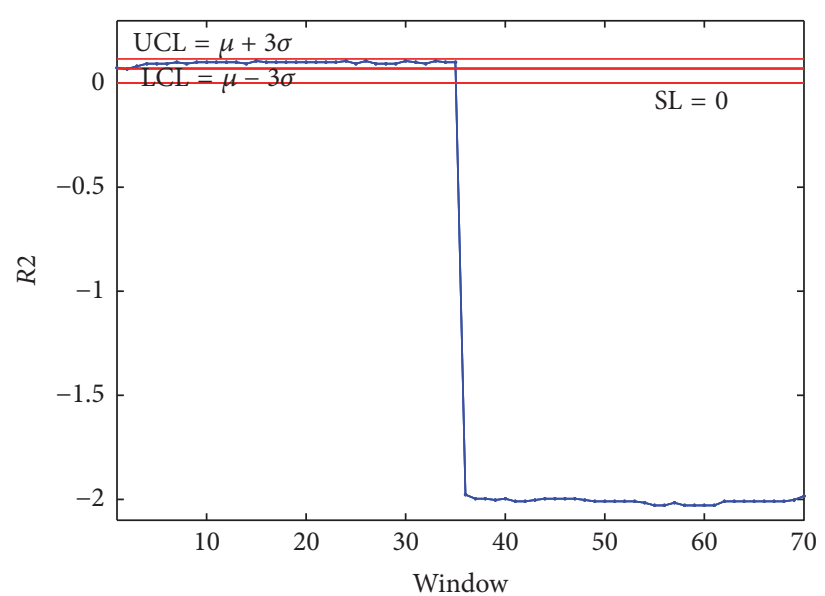

(b)

FIGURE 7: Stability monitoring with a process change $G_{p}=\left(0.6-0.3 z^{-1}\right) /\left(1-0.7 z^{-1}\right) \rightarrow G_{p}=\left(0.6 z^{-1}-0.3 z^{-2}\right) /\left(1-0.7 z^{-1}\right)$.

\section{Acknowledgments}

This work was supported by the National Natural Science Foundation of China under Grants nos. 61603347, 61603346, and 61603348 and the Doctor Startup Funds Foundation of Zhengzhou University of Light Industry under Grants nos. 2015BSJJ024 and 2015BSJJ026.

\section{References}

[1] N. Lu, Y. Yao, F. Gao, and F. Wang, "Two-dimensional dynamic PCA for batch process monitoring," AIChE Journal, vol. 51, no. 12, pp. 3300-3304, 2005.

[2] J. Chen and C.-K. Kong, "Performance assessment for iterative learning control of batch units," Journal of Process Control, vol. 19, no. 6, pp. 1043-1053, 2009.

[3] S. Wei, J. Cheng, and Y. Wang, "Data-driven two-dimensional LQG benchmark based performance assessment for batch processes under ILC," in Proceedings of the 9th IFAC Symposium on Advanced Control of Chemical Processes (ADCHEM '15), pp. 291-296, Whistler, Canada, June 2015.

[4] J. Shi, F. Gao, and T.-J. Wu, "Robust iterative learning control design for batch processes with uncertain perturbations and initialization," AIChE Journal, vol. 52, no. 6, pp. 2171-2187, 2006.

[5] Y. Wang, Y. Yang, and Z. Zhao, "Robust stability analysis for an enhanced ILC-based PI controller," Journal of Process Control, vol. 23, no. 2, pp. 201-214, 2013.

[6] D. D. Siljak, "Stability criteria for two-variable polynomials," Institute of Electrical and Electronics Engineers. Transactions on Circuits and Systems, vol. 22, no. 3, pp. 185-189, 1975.

[7] Y. Yao and F. R. Gao, "Batch process monitoring in score space of two dimensional dynamic Principal Component Analysis (PCA)," Industrial \& Engineering Chemistry Research, vol. 46, no. 24, pp. 8033-8043, 2007.

[8] B. Aksasse and L. Radouane, "Two-dimensional autoregressive (2-D AR) model order estimation," IEEE Transactions on Signal Processing, vol. 47, no. 7, pp. 2072-2077, 1999.
[9] Y. Yao and F. R. Gao, "Statistical monitoring and fault diagnosis of batch processes using two-dimensional dynamic information," Industrial and Engineering Chemistry Research, vol. 49, no. 20, pp. 9961-9969, 2010.

[10] H. Zou, "The adaptive lasso and its oracle properties," Journal of the American Statistical Association, vol. 101, no. 476, pp. 14181429, 2006.

[11] Y. Wang, Y. Yao, Y. Zheng, and D. S. Wong, "Monitoring of within batch and batch-to-batch dynamics using adaptive LASSO," International Journal of System Control and Information Processing, vol. 1, no. 4, 2015.

[12] G. E. Box, G. M. Jenkins, G. Reinsel, and G. M. Ljung, Time Series Analysis: Forecasting and Control, Wiley Series in Probability and Statistics, John Wiley \& Sons, Hoboken, NJ, USA, 5th edition, 2015.

[13] A. Kanellakis, S. Tzafestas, and N. Theodorou, "Stability tests for 2-D systems using the schwarz form and the inners determinants," IEEE Transactions on Circuits and Systems, vol. 38, no. 9, pp. 1071-1077, 1991.

[14] E. I. Jury, "Theory and applications of the inners," IEEE Transaction on Automatic Control, vol. 63, no. 7, pp. 1044-1069, 1971. 


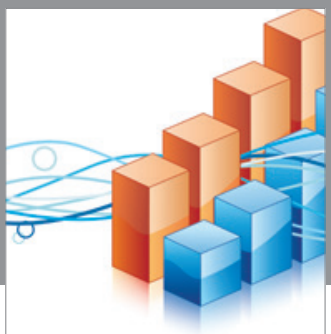

Advances in

Operations Research

vatem alat4

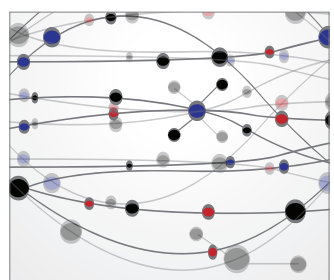

\section{The Scientific} World Journal
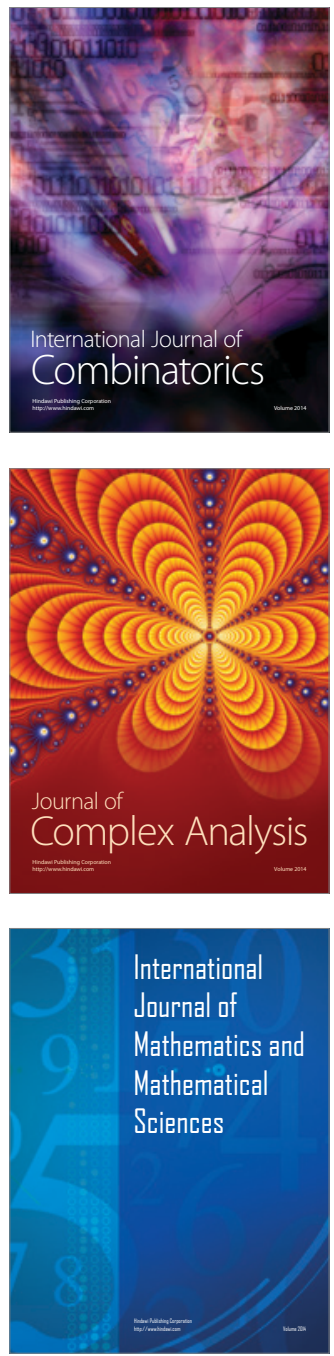
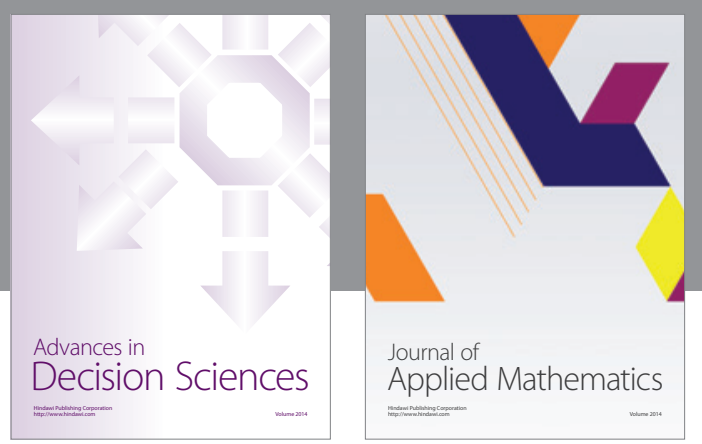

Algebra

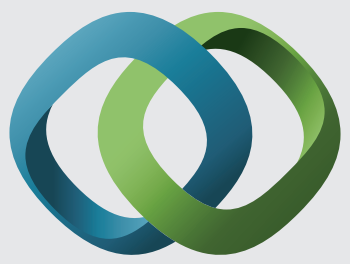

\section{Hindawi}

Submit your manuscripts at

https://www.hindawi.com
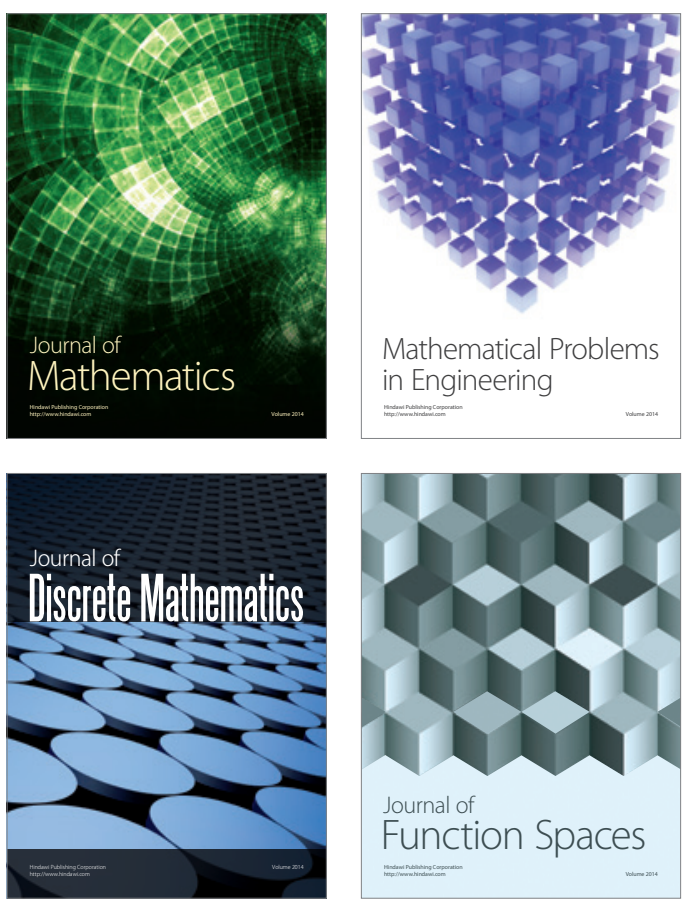

Mathematical Problems in Engineering
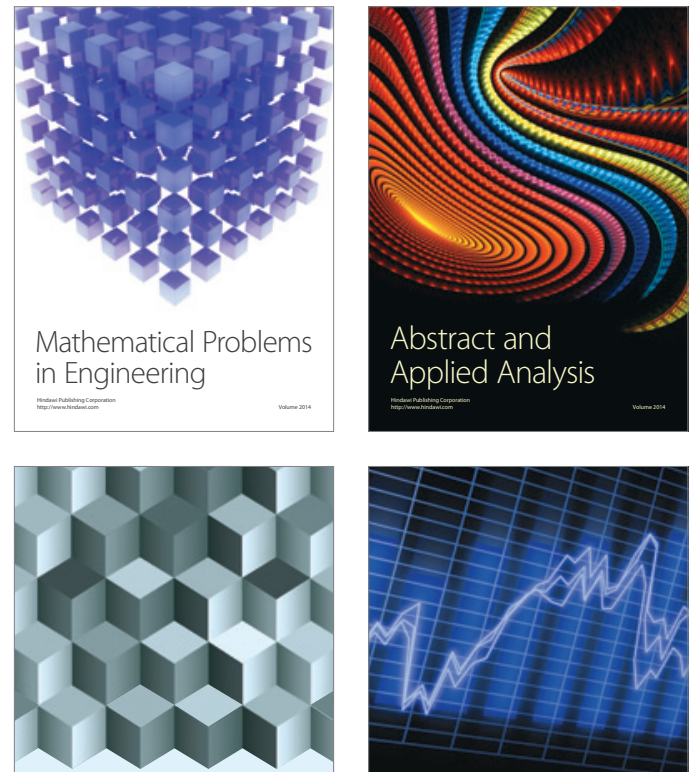

Journal of

Function Spaces

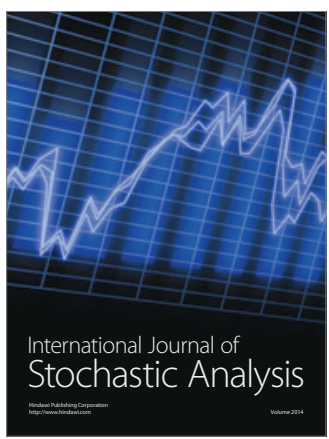

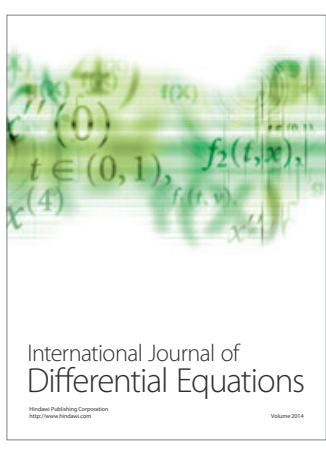
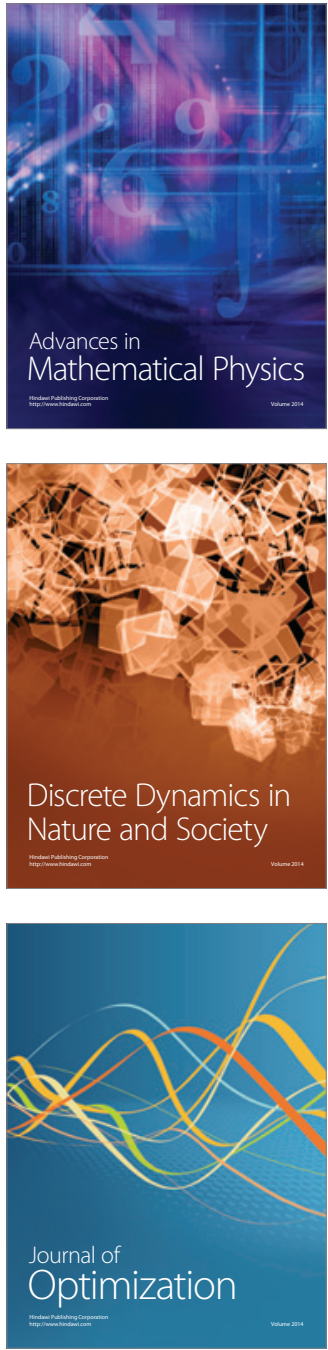